\title{
Dynamic hormone control of stress and
}

\section{fertility}

Eder Zavala ${ }^{1,2, \ddagger}$, Margaritis Voliotis ${ }^{3, \ddagger}$, Tanja Zerenner ${ }^{3, \ddagger}$, Joël Tabak $^{4}$, Jamie

J Walker ${ }^{3,5}$, Xiao Feng Li ${ }^{6}$, John R Terry ${ }^{1,2}$, Stafford L Lightman ${ }^{5}$, Kevin

O’Byrne $^{6}$, Krasimira Tsaneva-Atanasova ${ }^{3,7, *}$

${ }^{1}$ Centre for Systems Modelling and Quantitative Biomedicine, University of

Birmingham, Birmingham, United Kingdom

${ }^{2}$ Institute of Metabolism and Systems Research, College of Medical and Dental

Sciences, University of Birmingham, Birmingham, United Kingdom

${ }^{3}$ EPSRC Centre for Predictive Modelling in Healthcare, Living Systems Institute,

College of Engineering, Mathematics and Physical Sciences, University of Exeter,

Exeter, United Kingdom

${ }^{4}$ Institute of Biomedical and Clinical Science, College of Medicine and Health,

University of Exeter, Exeter, United Kingdom

${ }^{5}$ Henry Wellcome Laboratory for Integrative Neuroscience and Endocrinology,

Translational Health Sciences, Bristol Medical School, University of Bristol, Bristol,

United Kingdom

${ }^{6}$ Department of Women and Children's Health, School of Life Course Sciences,

King's College London, London, United Kingdom

${ }^{7}$ Department of Bioinformatics and Mathematical Modelling, Institute of Biophysics and Biomedical Engineering, Bulgarian Academy of Sciences, Sofia, Bulgaria

$\ddagger$ Equal contribution

Correspondence*:

Krasimira Tsaneva-Atanasova

K.Tsaneva-Atanasova@exeter.ac.uk 


\section{ABSTRACT}

3 Neuroendocrine axes display a remarkable diversity of dynamic signalling processes relaying

4 information between the brain, endocrine glands, and peripheral target tissues. These dynamic

5 processes include oscillations, elastic responses to perturbations, and plastic long term changes

6 observed from the cellular to the systems level. While small transient dynamic changes can be

7 considered physiological, larger and longer disruptions are common in pathological scenarios

8 involving more than one neuroendocrine axes, suggesting that a robust control of hormone

9 dynamics would require the coordination of multiple neuroendocrine clocks. The idea of apparently

10 different axes being in fact exquisitely intertwined through neuroendocrine signals can be

11 investigated in the regulation of stress and fertility. The stress response and the reproductive

12 cycle are controlled by the Hypothalamic-Pituitary-Adrenal (HPA) axis and the Hypothalamic-

13 Pituitary-Gonadal (HPG) axis, respectively. Despite the evidence surrounding the effects of

14 stress on fertility, as well as of the reproductive cycle on stress hormone dynamics, there is

15 a limited understanding on how perturbations in one neuroendocrine axis propagate to the

16 other. We hypothesize that the links between stress and fertility can be better understood by

17 considering the HPA and HPG axes as coupled systems. In this manuscript, we investigate

18 neuroendocrine rhythms associated to the stress response and reproduction by mathematically

19 modelling the HPA and HPG axes as a network of interlocked oscillators. We postulate a network architecture based on physiological data and use the model to predict responses to stress

21 perturbations under different hormonal contexts: normal physiological, gonadectomy, hormone

22 replacement with estradiol or corticosterone (CORT), and high excess CORT (hiCORT) similar to hypercortisolism in humans. We validate our model predictions against experiments in rodents,

24 and show how the dynamic responses of these endocrine axes are consistent with our postulated network architecture. Importantly, our model also predicts the conditions that ensure robustness of fertility to stress perturbations, and how chronodisruptions in glucocorticoid hormones can health. 
Keywords: CORT, fertility, GnRH pulse generator, glucocorticoids, hormone dynamics, hypercortisolism, KNDy network, mathematical

model, stress

\section{INTRODUCTION}

A robust dynamic interplay between body rhythms is essential to sustain healthy states. This requires

the coordination of several regulatory systems spanning multiple levels of organisation, from molecular,

to cellular, to the whole organism. Neuroendocrine axes are the perfect example of such interlockedregulatory systems controlling body rhythms, with the brain decoding circadian and stress inputs as well as integrating feedback signals from endocrine organs. The hypothalamic-pituitary-adrenal (HPA) axis and the hypothalamic-pituitary-gonadal (HPG) axis are the major neuroendocrine systems underpinning stress and fertility, respectively. These axes control a range of hormonal and neural activity rhythms exhibiting ultradian $(<24 \mathrm{~h})$, circadian $(\sim 24 \mathrm{~h})$ and infradian $(>24 \mathrm{~h})$ periodicity [1], as well as responses to environmental, biological and behavioural perturbations. For example, the HPA axis uses feedback loops to regulate stress responses while sustaining ultradian and circadian glucocorticoid (CORT) rhythms [2, 3]. On the other hand, the HPG axis controls infradian oscillations of reproductive hormones secreted in response to changes in the ultradian frequency of gonadotropin-releasing hormone $(\mathrm{GnRH})$. GnRH secretion is controlled by a hypothalamic pulse generator (PG) [4], which is in turn modulated by reproductive hormones (Fig. 1A). Mathematical modelling has significantly contributed to our understanding of the origin of this rhythmic behaviour [3, 4, 5, 6, 7], as well as the ability of these systems to respond to perturbations.

In recent years, animal models have greatly enhanced our understanding of how specific perturbations in the HPA axis dynamics affect the HPG axis [8, 9, 10, 11], as well as how the activity of the HPG axis can in turn modulate the dynamics of the HPA axis [12, 13, 14, 15]. Furthermore, human studies have shown

51 how glucocorticoid excess can have profound effects on the menstrual cycle [16, 17, 18]. Many studies have shown the links between stress and fertility [19, 20, 21, 22] and provided evidence of cross-regulatory interactions between the HPA and HPG axes [23, 24]. However, there is still a limited understanding of whether and how the HPA and HPG axes coordinate their hormone rhythms, how perturbations to one axis impact upon the other, what makes their dynamics robust to such perturbations, and in what circumstances chrono-disruptions can lead to disease. 


\section{Zavala et al.}

Modelling stress and fertility

\section{MODEL AND METHODS}




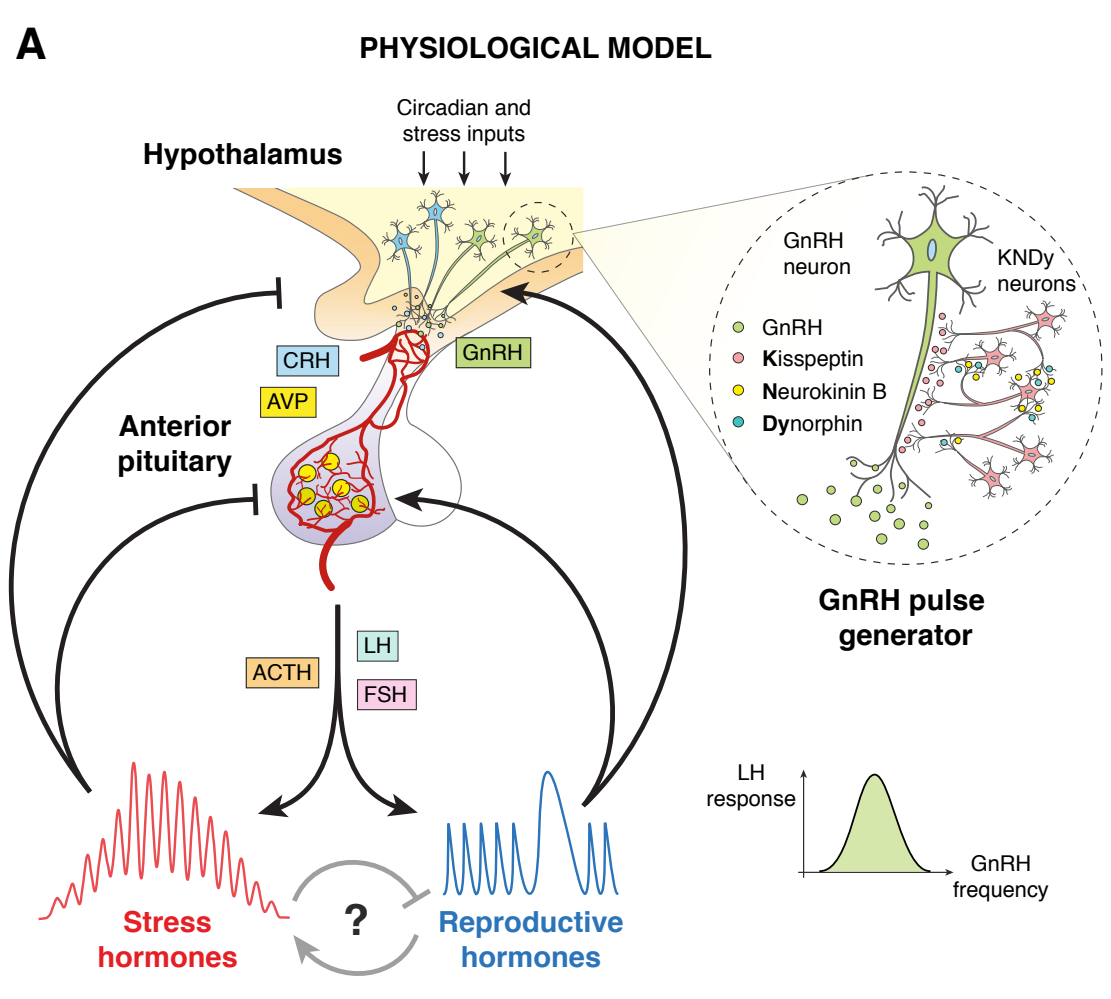

B

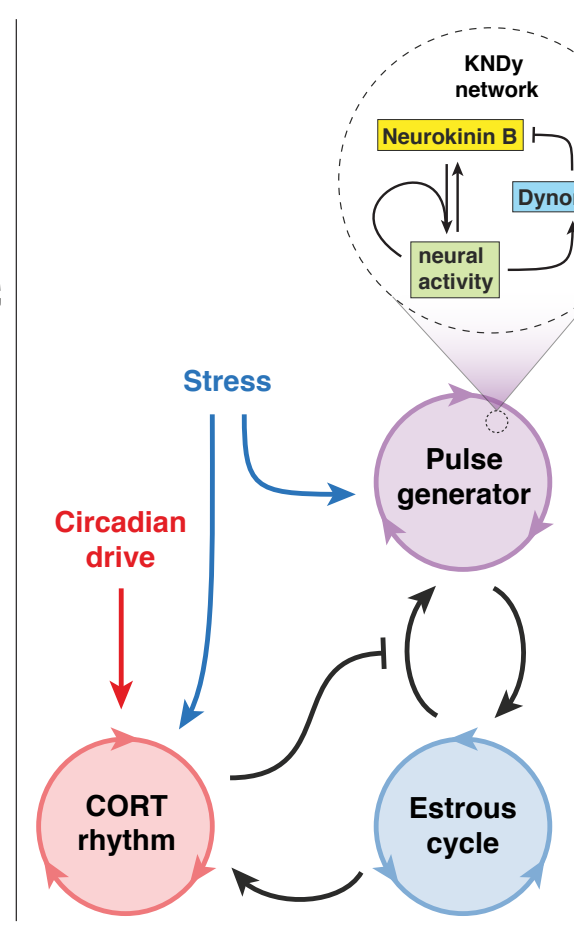

Figure 1. Pictorial representation of the model. (A) Physiological model of the stress and reproductive neuroendocrine axes controlling ultradian, circadian and infradian hormone oscillations. Includes the KNDy neuronal network controlling the GnRH pulse generator. Adapted from [5]. (B) Network model of the systems-level cross-regulation between glucocorticoid (CORT) rhythms, the hypothalamic GnRH pulse generator and the estrous cycle, subject to stress and circadian inputs. Includes a mean-field model of the KNDy network from [4].

84 Material.

Circadian cycle: A fixed period hypothalamic oscillator to control the circadian rhythm of CORT:

$$
\frac{d}{d t} \varphi_{H}=\omega_{H_{0}}
$$

where $\varphi_{H}$ is the hypothalamic phase and $\omega_{H_{0}}$ is the natural frequency of the hypothalamic circadian drive.

CORT oscillator: Accounts for CORT ultradian oscillations originating from the pituitary-adrenal

88 feedback loop [3]. Its dynamics can be affected by stressors, exogenous CORT, and the estrous cycle. The

89 phase $\varphi_{C}$ is given by:

$$
\frac{d}{d t} \varphi_{C}=\omega_{C_{0}}-\alpha s\left(\varphi_{H}\right)
$$


90 where $\omega_{C_{0}}$ is the natural frequency of CORT ultradian oscillations, $s\left(\varphi_{H}\right)$ is a function accounting for a

91 transient acute stressor (equal to zero in the absence of stress), and $\alpha$ is a scaling factor accounting for how

92 strongly such stressor temporarily disrupts CORT ultradian rhythmicity. The amplitude $A_{C}$ is given by:

$$
\frac{d}{d t} A_{C}=f_{H}\left(\varphi_{H}\right) \frac{A_{E}^{n}}{A_{E}^{n}+K_{E}^{n}}-A_{C}+s\left(\varphi_{H}\right)
$$

93 where $f_{H}\left(\varphi_{H}\right)$ is a function representing hypothalamic circadian modulation. $A_{E}$ is the amplitude of the

94 estrous cycle (representative of the level of sex steroids) which modulates $A_{C}$ through a Hill type function

95 with coefficient $n$ and half-maximum constant $K_{E}$.

Pulse generator: Accounts for the activity of the GnRH pulse generator. Its frequency is modulated by

97 stressors, CORT levels, and the activity of the KNDy network [4], which is in turn influenced by the phase

98 of the estrous cycle. The phase $\varphi_{P G}$ is given by:

$$
\frac{d}{d t} \varphi_{P G}=\omega_{P G}
$$

99 where $\omega_{P G}$ denotes the varying frequency of the pulse generator. This is given by:

$$
\frac{d}{d t} \omega_{P G}=\omega_{P G_{m}} f_{K}(N, D, \widetilde{C})-\omega_{P G}
$$

100 where $\omega_{P G_{m}}$ is the maximum frequency of the pulse generator and $f_{K}(N, D, \widetilde{C})$ is a function accounting 101 for the regulation from the KNDy network and CORT. Equations for the excitatory ( $N$; e.g., Neurokinin B 102 and glutamate) and inhibitory ( $D$; e.g., Dynorphin) signals regulating the frequency of the KNDy network, 103 and the slow genomic CORT effects $(\widetilde{C})$ are given in the Supplementary Material.

Estrous cycle: Accounts for the activity of the reproductive cycle. The phase $\varphi_{E}$ is given by:

$$
\frac{d}{d t} \varphi_{E}=\omega_{E_{m}} f_{P G}\left(\omega_{P G}\right),
$$


where $f_{P G}\left(\omega_{P G}\right)$ is a function accounting for the effects of the pulse generator and $\omega_{E_{m}}$ is the maximum frequency of the estrous cycle. The amplitude $A_{E}$ is given by:

$$
\frac{d}{d t} A_{E}=\varepsilon+\beta f_{E}\left(\varphi_{E}\right)-A_{E}
$$

\section{RESULTS}

where $\varepsilon$ is the basal activity of the estrous cycle, $f_{E}\left(\varphi_{E}\right)$ is a function representing the effects of the estrous cycle, and $\beta$ is a scaling factor accounting for the strength of such effects.

\subsection{Computer simulations and parameter estimation}

To simplify our analysis, CORT oscillations were normalised to the maximum levels observed in physiological conditions. That is, the CORT amplitude, which is modulated by the circadian drive, spans the range between 0 and 1 unless stressors or exogenous CORT act upon it. Similarly, the activity of the PG was represented by normalised oscillations, with a frequency that changes periodically according to the different stages of the estrous cycle. The model equations were numerically solved and analysed in MATLAB R2020a using ode45 routines. Details of the mathematical model development and parameter values are described in the Supplementary Material. The model parameters were estimated from the literature where available and manually calibrated to reproduce experimental observations of CORT and reproductive rhythms in rodents.

\subsection{Normal physiological HPA and HPG rhythms}

We calibrate the model parameters to reproduce physiological HPA and HPG rhythms observed in rats [1]. Accordingly, our model simulates CORT oscillations with a 75 min period, while the amplitude of these ultradian pulses is modulated in a circadian manner, reaching a maximum at the start of the dark period (Fig. 2A). Furthermore, one full estrous cycle lasts approximately 4 days, matching the average cycle length measured in rats [30]. A recent study using fibre photometry calcium imaging from arcuate kisspeptin neurons in mice revealed the dynamic modulation of GnRH pulse frequency along the estrous cycle [31]. Following these findings, the activity of the PG in the model remains inhibited (below 1 pulse/hr) during the post-ovulatory, estrous phase, rises steeply at the start of metestrus, and levels off at 2 pulses/hr for the rest of the cycle (Fig. 2B). 

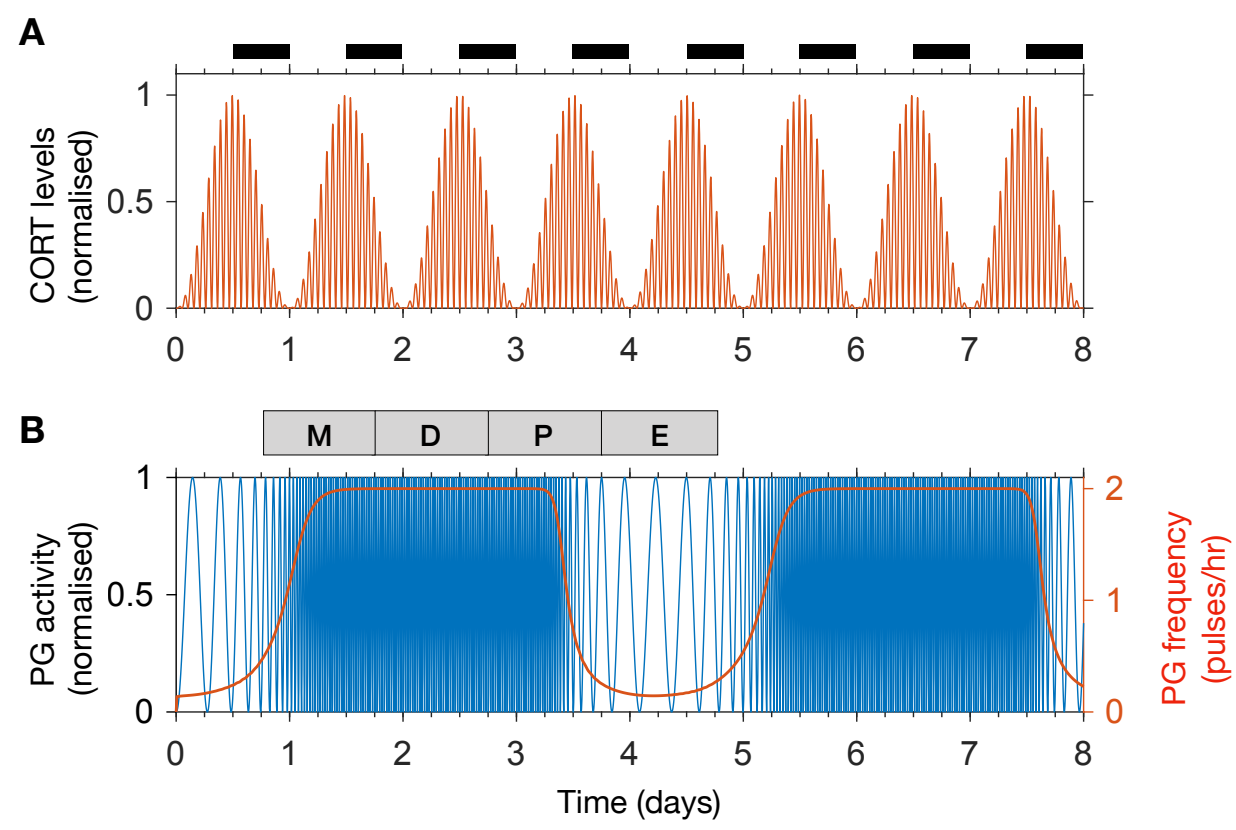

Figure 2. The model reproduces physiological rhythms in the HPA and HPG axis. (A) Normalised CORT levels as a function of time. The light-dark cycle is represented with intermittent black bars on the top. (B) Normalised pulse generator activity (blue) and pulse generator frequency (red) as a function of time. The phases of the estrous cycle are marked on the top: estrus (E); metestrus (M); diestrus (D); and proestrus (P).

\subsection{Recovery of CORT dynamics following ovariectomy}

Previous findings suggest that gonadal steroids are integral to the increased CORT levels seen in females compared to males. This has been demonstrated by showing the effects of oestrogen replacement in recovering physiological CORT levels following ovariectomy in rats [12]. We investigate the dynamic $(\mathrm{OVX})$ and its restitution following $17 \beta$-oestradiol $\left(\mathrm{E}_{2}\right)$ replacement. In the model, this is achieved by replacing the influx term in the right hand side of Eq. 7by a constant term representing a drop in $\mathrm{E}_{2}$ levels following OVX (causing $A_{E}$ to drop down to a constant level of $2 \%$ from the estrous peak) and by replacing the periodic sensitivity of the KNDy network to the estrous phase by a constant low value (Supplementary Material). The model predicts a drop in CORT levels down to $\sim 30 \%$ from its physiological value without loss of circadian or ultradian CORT rhythmicity while keeping the PG frequency at a high constant value of 2 pulses/hr (Fig. 3A). We then simulated the effects of an $\mathrm{E}_{2}$ pellet on OVX rats by increasing the constant value of the influx term in the right hand side of Eq. 7)(98\% from physiological $\left.A_{E}\right)$ and increasing the sensitivity of the KNDy network to the estrous phase $\left(\varphi_{E}\right)$ by a constant value 3 (Supplementary Material). In agreement with [12], the model predicts recovery of physiological CORT 
144 levels without loss of circadian or ultradian CORT rhythmicity while marginally reducing the PG frequency 145 just below 2 pulses/hr (Fig. 3B).
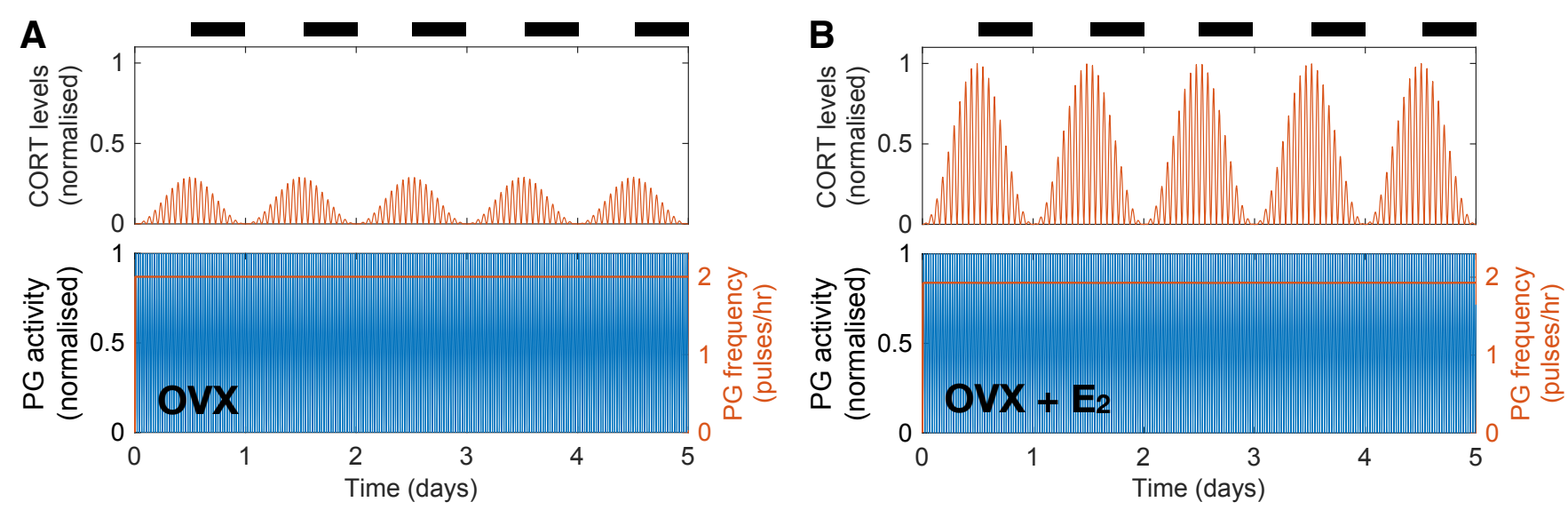

Figure 3. The model explains how $E_{2}$ replacement recovers physiological CORT levels in OVX rats. (A) Simulated OVX reduced CORT oscillations down to $\sim 30 \%$ of the maximum physiological levels while keeping a constant high PG activity. (B) Simulated OVX+E 2 recovered CORT oscillations to physiological levels while keeping a constant high PG activity.

\subsection{Estradiol-mediated inhibition of HPG dynamics by high CORT doses}

In a recent study, Kreisman and co-workers [8] investigated the effect of chronic CORT administration on

LH pulsatility and demonstrated the importance of gonadal steroid hormones in mediating the inhibitory effect of CORT on the HPG axis. The study showed that a pellet delivering a high dose of CORT over 48 hrs in OVX mice has no effect on LH pulsatility, whereas a significant reduction of LH pulse frequency is observed in OVX animals treated with a $17 \beta$-estradiol silastic implant $\left(\mathrm{OVX}+\mathrm{E}_{2}\right)$. In our model, we accounted for the $\mathrm{OVX}$ and $\mathrm{OVX}+\mathrm{E}_{2}$ scenarios as described in the previous section, while the constantly high CORT levels were achieved by replacing the effective CORT levels modulating the KNDy network by a constant high value estimated from [8] (see Supplementary Material).

Figure 4 illustrates the differential effect of chronically elevated CORT levels on the GnRH pulse generator frequency in OVX versus $\mathrm{OVX}+\mathrm{E}_{2}$ animals. In the case of OVX animals, elevated CORT levels do not alter the frequency of the pulse generator, whereas in OVX animals treated with estradiol the frequency is halved for as long as CORT levels are elevated. This effect is linked to the modulation of the GnRH pulse generator by gonadal steroids, which sensitise the system to inhibitory signals such as CORT or acute stressors as we show below (Fig. $4 \mathrm{~B})$. 

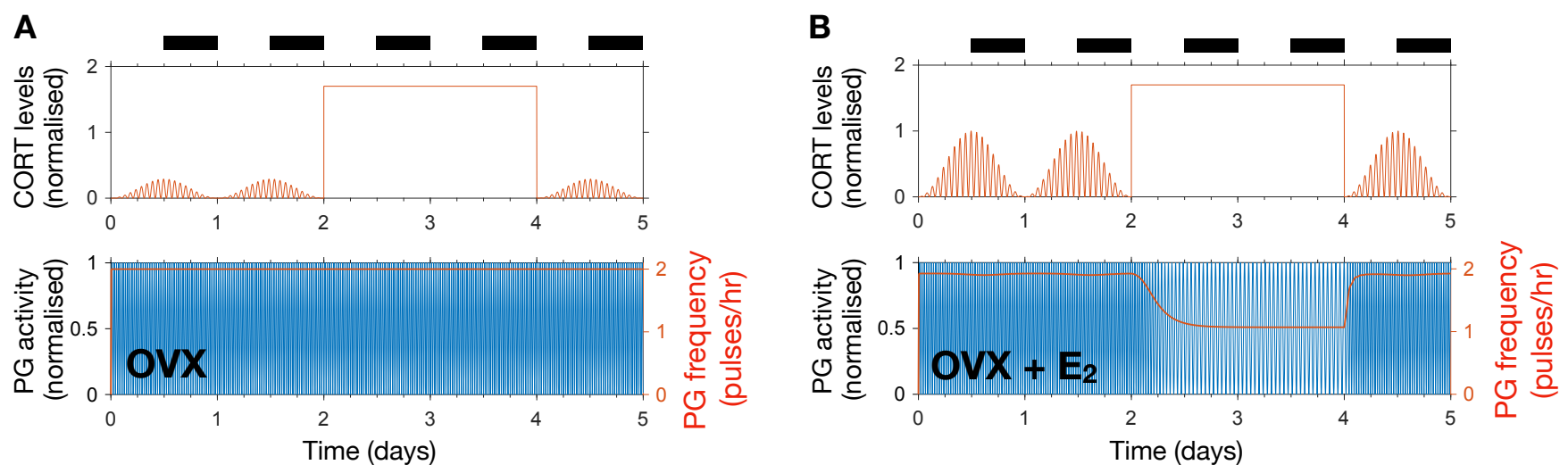

Figure 4. The model reproduces estradiol-mediated inhibition of PG activity following high doses of CORT. (A) High exogenous CORT over 48 hrs does not affect the PG dynamics in OVX mice. (B) In the presence of estradiol, high CORT doses temporarily reduce PG activity in OVX mice.

\subsection{Acute stress effects on the HPA and HPG axes depend on the estrous cycle phase}

To study the effect of acute stress on the dynamics of the HPA and HPG axes, we extend the model to include transient stress-related neuronal inputs affecting both axes [11]. In our model, we account for these transient inputs by simulating a $2 \mathrm{hr}$ square pulse of amplitude 1, equivalent to a restraint stressor causing a CORT increase from its circadian nadir up to its circadian peak [32]. The stressor affects the phase and amplitude of the CORT rhythm (function $s\left(\varphi_{H}\right)$ in Eqs. 2 and 3) as well as the frequency of the GnRH pulse generator (function $f_{K}(N, D, \widetilde{C})$ in Eq. 5 and Supplementary Material).

Figures $5 \mathrm{~A}$ and $5 \mathrm{~B}$ illustrate the effect that $2 \mathrm{hr}$ of stress activation has on the dynamics of the HPA and HPG axes when applied at different times along the cycle. Both CORT and GnRH pulse generator responses are dependent on the timing of the input pulse (Fig. 5C). The amplitude of the CORT response shows a circadian dependency with stressors delivered during the circadian peak eliciting a stronger response. The GnRH pulse generator frequency response to acute stressors depends on the phase of the estrous cycle. In diestrus phases, with little or no effect during the mid-cycle phase. This differential effect of acute stress on the frequency of the GnRH pulse generator activity highlights the cycle dependent modulation of the pulse generator dynamics, which makes the pulse generator more robust to perturbations in the diestrus 

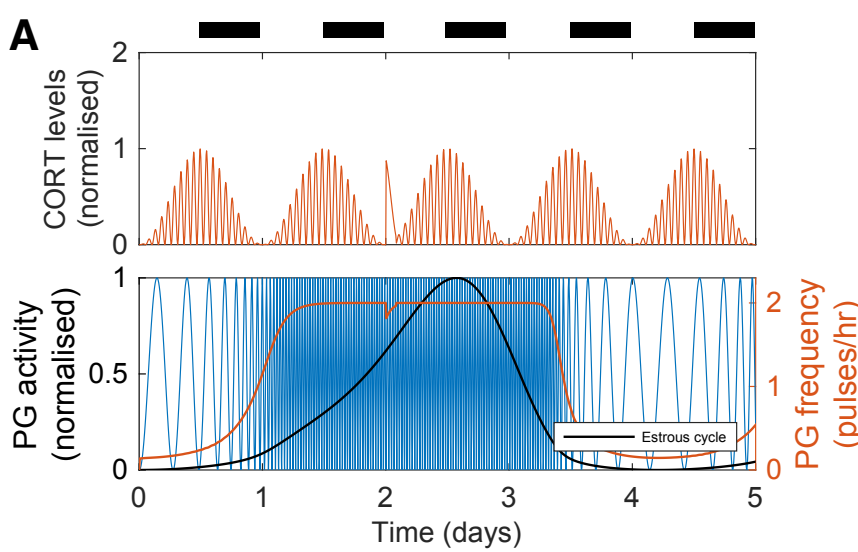

C
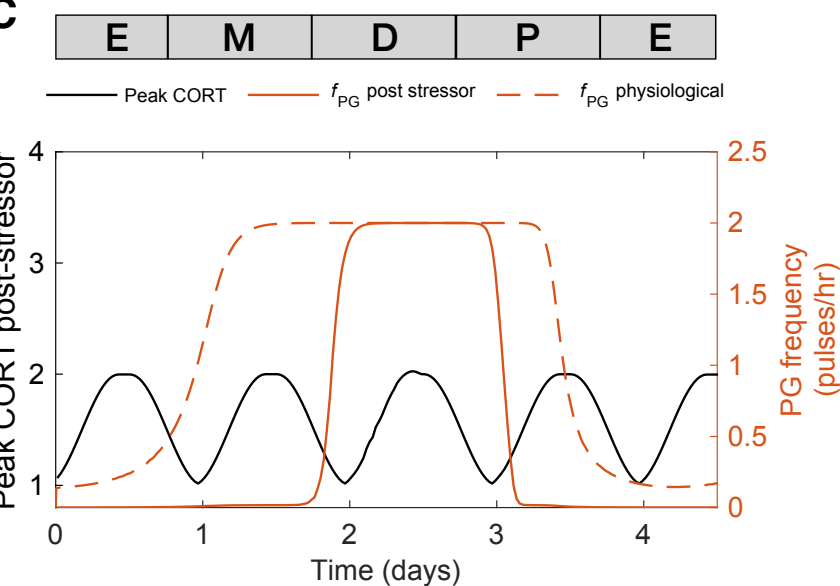
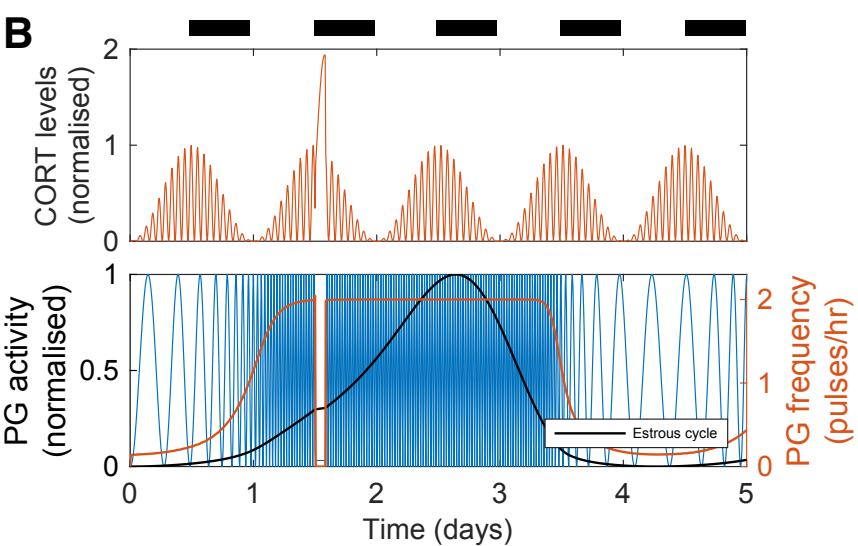

D

2D state space describing pulse generator dynamics

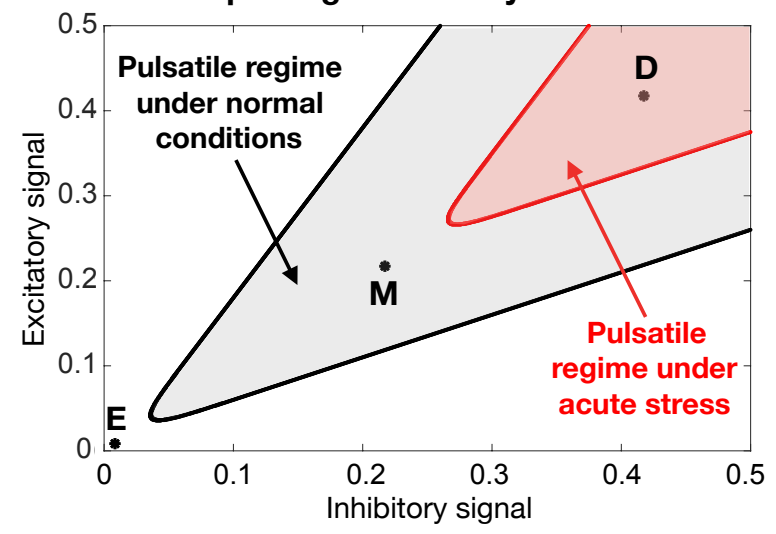

Figure 5. The effect of acute stress on the dynamics of the HPA and HPG axes. (A-B) CORT levels and PG activity in response to a transient ( $2 \mathrm{hr}$ long) stressor initiated at two different times. (C) Peak CORT levels (black line) and mean PG frequency (continuous red line) elicited by a $2 \mathrm{hr}$ long acute stressor as a function of the time at which the stressor arrives during the estrous cycle. The PG frequency without any stress perturbation is shown for comparison (dashed red line). (D) State space diagram describing the effect of acute stress on the dynamics of the pulse generator. Points mark different stages along the estrus cycle: estrus midpoint (E); metestrus midpoint (M); and diestrus midpoint (D). The shaded gray area denotes the region of the state space corresponding to frequencies above 1 pulse/hr under normal physiological conditions. Acute stress shrinks this region (red shaded area), but the dynamics of the pulse generator maintains robustness to perturbations during the diestrus phase.

\subsection{CORT excess increases the length of the estrous cycle and modulates responses} to acute stressors

Last, we used the model to predict the effects of high excess CORT (hiCORT) — mimicking levels

expected to be observed in people with hypercortisolism— on the estrous cycle. To do this, we considered

the increase in baseline and maximum CORT amplitude with respect to physiological levels in humans

[33] and implemented the equivalent increase ratios for our simulations of CORT dynamics in rodents

184 (Supplementary Material). Evidence from high frequency sampling in humans shows hypercortisolism is 185 associated with a reduction in the ultradian period of CORT oscillations [34]. Accordingly, we also adjusted 


\section{Zavala et al.}

Modelling stress and fertility

186

187

188

189

190

191

192

193

194

195

196

197

198

199

200

201

202

\section{DISCUSSION}

We developed and studied a mathematical model that integrates components of the stress and reproductive

this parameter when modelling hiCORT, while keeping circadian oscillations and all other parameters unchanged. Our simulations predict an increase in the period of the estrous cycle from a physiological value of $\mathrm{T}_{\text {phys }}=4.2$ days up to $\mathrm{T}_{h i C}=5.1$ days under hiCORT, which is equivalent to a $\sim 21 \%$ increase in the estrous cycle length (Fig. 6A and C).

We then used the model to investigate the transient changes in the GnRH pulse generator frequency and CORT amplitude elicited by exogenous acute stressors under physiological conditions and hiCORT. In particular, we look at the effects of the timing of stressors within the estrous cycle. To do this, we calculated frequency and amplitude response curves by simulating a $2 \mathrm{hr}$ long stressor elicited at different stages across the estrous cycle using $30 \mathrm{~min}$ time steps. In the physiological scenario (Fig. 6B), the model predicts that acute stressors suppress PG activity during most of the estrous cycle except during the diestrus and early proestrus phases. These stressors also elicit an increase in peak CORT levels to a range between 1 and 2. While the model under the hiCORT scenario predicts a similar behaviour, the region where PG activity remains unaffected by acute stressors is reduced and delayed by about half a day compared to its physiological counterpart (Fig. 6D and Fig. S2). This is not surprising considering that the model also predicts that hiCORT prolongs the estrous cycle. Regarding the CORT response to stressors under hiCORT, our model predicts a $\sim 2$ to $\sim 3.5$ increase in CORT levels compared to the normal physiological scenario. This is due to a compounded effect of CORT surges over an excess CORT baseline.

axes at different spatial and temporal scales, from the molecular intricacies of the KNDy network, to GnRH and CORT oscillations, up to the estrous cycle (Fig. 11A). Previous mathematical models of the HPA and HPG axes either focus on a specific process within an axis, or consider them as a whole, but isolated from each other [6, 2, 3, 4]. In contrast, our model integrates these neuroendocrine axes by considering the complex interactions between them as a network of interlocked oscillators, hence enabling us to integrate different physiological observations and experiments into a single coherent theoretical framework and study the effect of transient perturbations on the overall dynamics. In particular, our model postulated a network architecture (Fig. 1B) that reflects physiological observations of ultradian and circadian CORT rhythms, as well as ultradian and infradian rhythms of the GnRH pulse generator (Fig. 2). The model reproduced the 
A
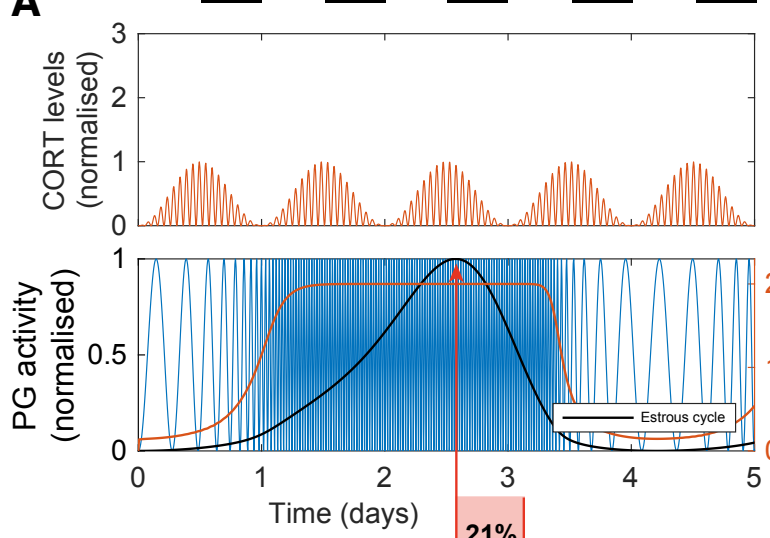

C
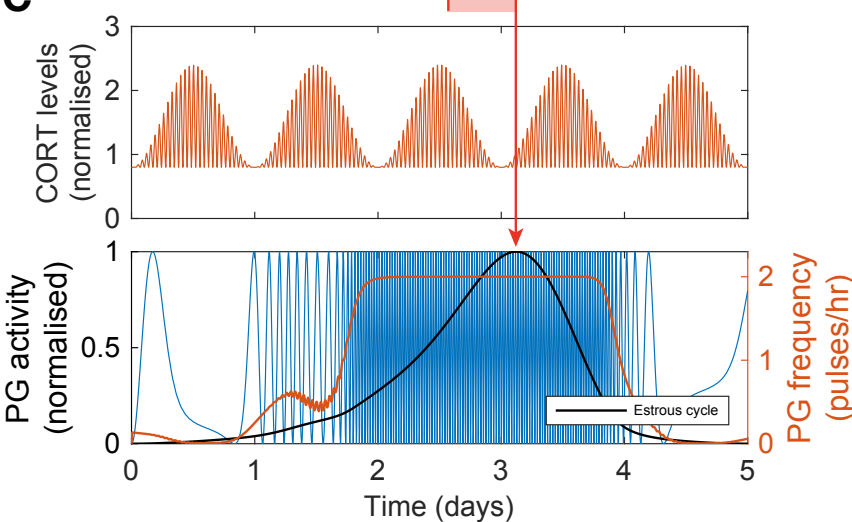
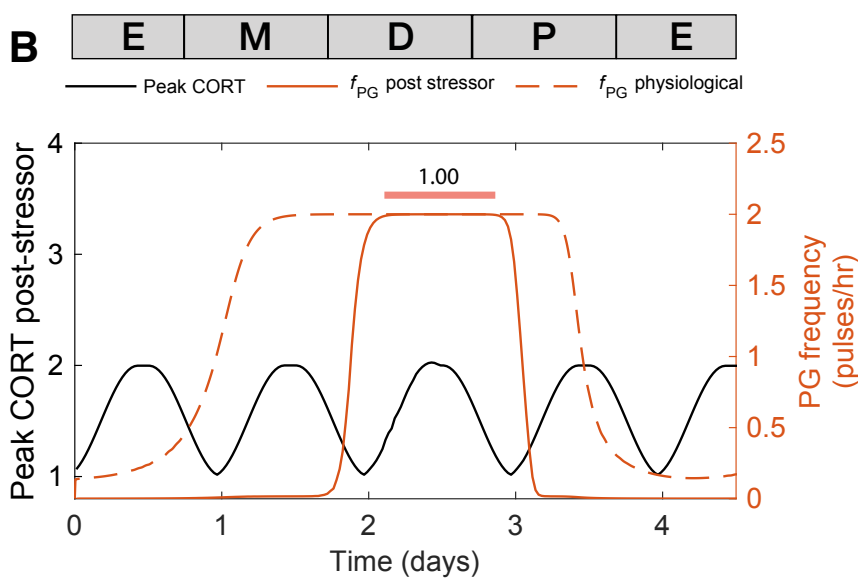

D
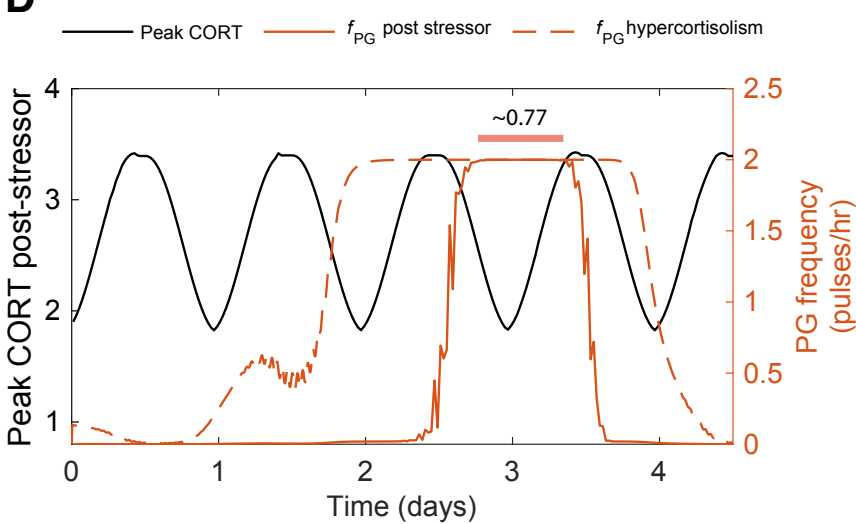

Figure 6. Enduring and transient dynamic changes under hiCORT. (A) CORT and PG rhythms in physiological conditions. (B) Mean PG frequency and maximum CORT levels elicited by 2 hr long hypothalamic stressors arriving at different times across the estrous cycle. (C) Under hiCORT, the range of CORT levels is increased and the estrous cycle peak is delayed by $\sim 21 \%$ compared to physiological values. (D) Mean PG frequency and maximum CORT elicited by $2 \mathrm{hr}$ long stressors under hiCORT. In this scenario, the period in which PG activity remains unchanged by stressors starts about half a day later and is shortened compared to normal physiological conditions.

effects of ovarian hormone removal (OVX) and restitution $\left(\mathrm{E}_{2}\right)$ on the HPA and HPG axes dynamics, both under physiological conditions [12] (Fig. 3) and under exogenous CORT excess [8] (Fig. 4).

In addition to these slow timescale perturbations, we also investigated the fast timescale perturbations

216 elicited by acute stressors. Our model predicted that exogenous stress perturbations not only cause transient 217 increases in CORT levels, but also transiently inhibit GnRH pulse generator activity with the magnitude 218 of this inhibition being dependent on the estrous and circadian phases (Fig. 5). This has important 219 implications about understanding how the timing of a stressor affects its ability to temporarily suppress the GnRH/LH ovulatory surge. According to our model, the pulse generator activity is robust to stress perturbations arriving between the diestrus and early proestrus stage, but is fragile to stressors arriving at estrus and metestrus stages (Fig. 5C). Uncovering the origin of this robustness is beyond the scope 


\section{Zavala et al.}

of our phenomenological model, but we can speculate that molecular mechanisms ensure the resilience of the reproductive cycle during the key stages leading to ovulation. While our model suggests that the GnRH/LH surge should be delayed under frequent exposure to stressors, if the exposure occurs too close to the proestrus stage then these resilience molecular mechanisms ensure the surge continues as normal and triggers ovulation [35, 36].

We also used the model to investigate the potential detrimental effects on fertility elicited by chronic

hypercortisolism. Our model predicted that hiCORT delays the increase in activity of the GnRH pulse generator, effectively prolonging the estrous cycle (Fig. 6A and C). While evidence suggests that HPA axis hyperactivity — and specifically, increased circulating glucocorticoids — are unlikely to be the sole mechanism behind stress-induced reproductive dysfunction [19], our simulations show the cycle length depends on the GnRH pulse generator's sensitivity to CORT (Fig. S1A). Thus, our model provides insight into how for example a hyper-sensitized HPG axis may explain amenorrhea secondary to high serum cortisol levels [16, 17, 18]. Interestingly, our model predicted that a period of robustness of the GnRH pulse generator in the presence stressors is preserved under hiCORT, albeit the robust period occurs about half a day later in the cycle and is shorter in duration. Our model simulations of pulse generator activity suggest that prolonging the estrous cycle as predicted under hiCORT arises from a combination of longer estrus and metestrus stages while diestrus and proestrus stages are shortened (Fig. 6B and D).

Our model considers essential features of HPA and HPG axes oscillators in a phenomenological way.

This approach facilitates the simulation of a range of physio- pathological scenarios, but inevitably imposes certain limitations. In contrast to mechanistic models where parameters are often linked to chemical kinetic rates, the parameters in our model represent natural and maximum frequencies, phase relationships, as well 245 approach limits the ability of the model to support discovery of specific molecular mechanisms, it can 246 be used to suggest experiments that explore systems level properties involving both neuroendocrine axes. 247 For example, evidence shows that in addition to exhibiting circadian and ultradian fluctuations, CORT 248 levels also change across the estrous cycle, with maximum levels around the diestrus and proestrus phase 249 [37, 38, 39]. While our model lacks the level of detail to describe the molecular mechanisms that underpin 250 estrous changes on CORT, it does suggest this is mediated by a regulatory link from the estrous oscillator to 


\section{Zavala et al.}

Modelling stress and fertility

\section{CONFLICT OF INTEREST STATEMENT}

\section{AUTHOR CONTRIBUTIONS}

\section{FUNDING}

This work was funded by the Medical Research Council (MRC) fellowship MR/P014747/1 (to E.Z.), MRC 
275 Biological Sciences Research Council (BBSRC) grant BB/S001255/1 (to M.V., X.F.L., K.O. and K.T.A.), 276 Engineering and Physical Sciences Research Council (EPSRC) grant EP/N014391/2 (to E.Z., M.V., T.Z., 277 J.T., J.J.W., J.T.R., S.L.L. and K.T.A.), and the Wellcome Trust Grant WT105618MA (to J.J.W., J.R.T. and K.T.A.).

\section{SUPPLEMENTAL DATA}

See annex Supplementary Material for auxiliary equations and coupling functions, tables of model parameter values, and supplementary figures.

\section{DATA AVAILABILITY STATEMENT}

\section{REFERENCES} request. $1226-1238$. E6466-E6474. 9738-9747. (2020) 110877.

The computer code generated through this study is the subject of current research and is available upon

[1] Walker J, Terry J, Tsaneva-Atanasova K, Armstrong S, McArdle C, Lightman S. Encoding and decoding mechanisms of pulsatile hormone secretion. Journal of neuroendocrinology 22 (2010)

[2] Spiga F, Zavala E, Walker JJ, Zhao Z, Terry JR, Lightman SL. Dynamic responses of the adrenal steroidogenic regulatory network. Proceedings of the National Academy of Sciences 114 (2017)

[3] Walker JJ, Terry JR, Lightman SL. Origin of ultradian pulsatility in the hypothalamic-pituitary-adrenal axis. Proceedings of the Royal Society B: Biological Sciences 277 (2010) 1627-1633.

[4] Voliotis M, Li XF, De Burgh R, Lass G, Lightman SL, O’Byrne KT, et al. The origin of gnrh pulse generation: An integrative mathematical-experimental approach. Journal of Neuroscience 39 (2019)

[5] Zavala E, Wedgwood KC, Voliotis M, Tabak J, Spiga F, Lightman SL, et al. Mathematical modelling of endocrine systems. Trends in Endocrinology \& Metabolism (2019).

[6] Clément F, Crépieux P, Yvinec R, Monniaux D. Mathematical modeling approaches of cellular endocrinology within the hypothalamo-pituitary-gonadal axis. Molecular and cellular endocrinology 
[7] Churilov AN, Milton J, Salakhova ER. An integrate-and-fire model for pulsatility in the neuroendocrine system;? a3b2 show [editpick]?;. Chaos: An Interdisciplinary Journal of Nonlinear Science 30 (2020) 083132.

[8] Kreisman M, McCosh R, Tian K, Song C, Breen K. Estradiol enables chronic corticosterone to inhibit pulsatile lh secretion and suppress kiss1 neuronal activation in female mice. Neuroendocrinology (2019).

[9] Li XF, Bowe JE, Mitchell JC, Brain SD, Lightman SL, O’Byrne KT. Stress-induced suppression of the gonadotropin-releasing hormone pulse generator in the female rat: a novel neural action for calcitonin gene-related peptide. Endocrinology 145 (2004) 1556-1563.

[10] Luo E, Stephens SB, Chaing S, Munaganuru N, Kauffman AS, Breen KM. Corticosterone blocks ovarian cyclicity and the lh surge via decreased kisspeptin neuron activation in female mice. Endocrinology 157 (2016) 1187-1199.

[11] Yang JA, Song CI, Hughes JK, Kreisman MJ, Parra RA, Haisenleder DJ, et al. Acute psychosocial stress inhibits lh pulsatility and kiss1 neuronal activation in female mice. Endocrinology 158 (2017) $3716-3723$.

[12] Seale J, Wood S, Atkinson H, Harbuz M, Lightman S. Gonadal steroid replacement reverses gonadectomy-induced changes in the corticosterone pulse profile and stress-induced hypothalamicpituitary-adrenal axis activity of male and female rats. Journal of neuroendocrinology 16 (2004) 989-998.

[13] Seale J, Wood S, Atkinson H, Bate E, Lightman S, Ingram C, et al. Gonadectomy reverses the sexually diergic patterns of circadian and stress-induced hypothalamic-pituitary-adrenal axis activity in male and female rats. Journal of neuroendocrinology 16 (2004) 516-524.

[14] Seale J, Wood S, Atkinson H, Lightman S, Harbuz M. Organizational role for testosterone and estrogen on adult hypothalamic-pituitary-adrenal axis activity in the male rat. Endocrinology 146 (2005) 1973-1982.

[15] Seale J, Wood S, Atkinson H, Harbuz M, Lightman S. Postnatal masculinization alters the hpa axis phenotype in the adult female rat. The Journal of physiology 563 (2005) 265-274.

[16] DING JH, SHECKTER CB, DRINKWATER BL, SOULES MR, BREMNER WJ. High serum cortisol levels in exercise-associated amenorrhea. Annals of internal medicine 108 (1988) 530-534. 


\section{Zavala et al.}

Modelling stress and fertility

[17] Saketos M, Sharma N, Santoro NF. Suppression of the hypothalamic-pituitary-ovarian axis in normal women by glucocorticoids. Biology of Reproduction 49 (1993) 1270-1276.

[18] Suh B, Liu J, Berga S, Quigley M, Laughlin G, Yen S. Hypercortisolism in patients with functional hypothalamic-amenorrhea. The Journal of Clinical Endocrinology \& Metabolism 66 (1988) 733-739.

[19] Herod SM, Dettmer AM, Novak MA, Meyer JS, Cameron JL. Sensitivity to stress-induced reproductive dysfunction is associated with a selective but not a generalized increase in activity of the adrenal axis. American Journal of Physiology-Endocrinology and Metabolism 300 (2011) E28-E36.

[20] Herod SM, Pohl CR, Cameron JL. Treatment with a crh-r1 antagonist prevents stress-induced suppression of the central neural drive to the reproductive axis in female macaques. American Journal of Physiology-Endocrinology and Metabolism 300 (2011) E19-E27.

[21] Kalantaridou S, Makrigiannakis A, Zoumakis E, Chrousos G. Stress and the female reproductive system. Journal of reproductive immunology 62 (2004) 61-68.

[22] McCosh RB, Breen KM, Kauffman AS. Neural and endocrine mechanisms underlying stress-induced suppression of pulsatile lh secretion. Molecular and cellular endocrinology 498 (2019) 110579.

[23] Acevedo-Rodriguez A, Kauffman A, Cherrington B, Borges C, Roepke T, Laconi M. Emerging insights into hypothalamic-pituitary-gonadal axis regulation and interaction with stress signalling. Journal of neuroendocrinology 30 (2018) e12590.

[24] Crofford LJ, Jacobson J, Young E. Modeling the involvement of the hypothalamicpituitary-adrenal and hypothalamic-pituitary-gonadal axes in autoimmune and stress-related rheumatic syndromes in women. Journal of Women's Health 8 (1999) 203-215.

[25] Phumsatitpong C, Moenter SM. Estradiol-dependent stimulation and suppression of gonadotropinreleasing hormone neuron firing activity by corticotropin-releasing hormone in female mice. Endocrinology 159 (2018) 414-425.

[26] Li X, Edward J, Mitchell J, Shao B, Bowes J, Coen C, et al. Differential effects of repeated restraint stress on pulsatile lutenizing hormone secretion in female fischer, lewis and wistar rats. Journal of neuroendocrinology 16 (2004) 620-627.

[27] Li XF, Bowe JE, Lightman SL, O’Byrne KT. Role of corticotropin-releasing factor receptor-2 in stress-induced suppression of pulsatile luteinizing hormone secretion in the rat. Endocrinology 146 (2005) 318-322. 
[28] Grachev P, Li X, Hu M, Li S, Millar RP, Lightman SL, et al. Neurokinin b signaling in the female rat: a novel link between stress and reproduction. Endocrinology 155 (2014) 2589-2601.

[29] Lehman MN, Coolen LM, Goodman RL. Minireview: kisspeptin/neurokinin b/dynorphin (kndy) cells of the arcuate nucleus: a central node in the control of gonadotropin-releasing hormone secretion. Endocrinology 151 (2010) 3479-3489.

[30] McClintock MK. Estrous synchrony: modulation of ovarian cycle length by female pheromones. Physiology \& behavior 32 (1984) 701-705.

[31] McQuillan HJ, Han SY, Cheong I, Herbison AE. Gnrh pulse generator activity across the estrous cycle of female mice. Endocrinology 160 (2019) 1480-1491.

[32] Kitchener P, Di Blasi F, Borrelli E, Piazza PV. Differences between brain structures in nuclear translocation and dna binding of the glucocorticoid receptor during stress and the circadian cycle. European journal of neuroscience 19 (2004) 1837-1846.

[33] Vagnucci AH. Analysis of circadian periodicity of plasma cortisol in normal man and in cushing's syndrome. American Journal of Physiology-Regulatory, Integrative and Comparative Physiology 236 (1979) R268-R281.

[34] Van Aken M, Pereira A, Van Thiel S, Van Den Berg G, Frolich M, Veldhuis JD, et al. Irregular and frequent cortisol secretory episodes with preserved diurnal rhythmicity in primary adrenal cushing's syndrome. The Journal of Clinical Endocrinology \& Metabolism 90 (2005) 1570-1577.

[35] Wagenmaker ER, Breen KM, Oakley AE, Tilbrook AJ, Karsch FJ. The estrous cycle of the ewe is resistant to disruption by repeated, acute psychosocial stress. Biology of reproduction 82 (2010) $1206-1215$.

[36] Wagenmaker ER, Moenter SM. Exposure to acute psychosocial stress disrupts the luteinizing hormone surge independent of estrous cycle alterations in female mice. Endocrinology 158 (2017) 2593-2602.

[37] Atkinson HC, Waddell BJ. Circadian variation in basal plasma corticosterone and adrenocorticotropin in the rat: sexual dimorphism and changes across the estrous cycle. Endocrinology 138 (1997) $3842-3848$.

[38] Carey M, Deterd C, De Koning J, Helmerhorst F, De Kloet E. The influence of ovarian steroids on hypothalamic-pituitary-adrenal regulation in the female rat. Journal of endocrinology 144 (1995) 311-321. 
[39] Pilorz V, Steinlechner S, Oster H. Age and oestrus cycle-related changes in glucocorticoid excretion and wheel-running activity in female mice carrying mutations in the circadian clock genes per1 and per2. Physiology \& behavior 96 (2009) 57-63.

389 [40] Young E, Korszun A. The hypothalamic-pituitary-gonadal axis in mood disorders. Endocrinology and Metabolism Clinics of North America 31 (2002) 63-78.

391 [41] Massey AJ, Campbell BK, Raine-Fenning N, Pincott-Allen C, Perry J, Vedhara K. Relationship between hair and salivary cortisol and pregnancy in women undergoing ivf. Psychoneuroendocrinology 74 (2016) 397-405.

394 [42] Kim DW, Zavala E, Kim JK. Wearable technology and systems modeling for personalized chronotherapy. Current Opinion in Systems Biology (2020).

396 [43] Li K, Urteaga I, Wiggins CH, Druet A, Shea A, Vitzthum VJ, et al. Characterizing physiological and symptomatic variation in menstrual cycles using self-tracked mobile-health data. npj Digital Medicine 3 (2020) 1-13. 